\title{
1 Computer simulations to investigate the 2 causes of T-wave notching
}

3 Loriano Galeotti ${ }^{\mathrm{a}}$ Peter Van Dam ${ }^{\mathrm{b}, \mathrm{c}}$ Lars Johannesen $^{\mathrm{a}, \mathrm{d}}$ Jose Vicente $^{\mathrm{a}, \mathrm{e}}$ David G Strauss $^{\mathrm{a}}$

$4 \quad{ }^{a}$ US Food and Drug Administration, Silver Spring, MD, USA

$5 \quad{ }^{b}$ Radboud University, Nijmegen, the Netherlands

$6 \quad{ }^{\mathrm{C}}$ UCLA, Los Angeles, CA, USA

$7{ }^{d}$ Department of Clinical Physiology, Karolinska Institutet and Karolinska University Hospital, Stockholm,

8 Sweden

$9 \quad{ }^{\mathrm{e}} \mathrm{BSICOS}$ Group, I3A, IIS Aragón, University of Zaragoza, Zaragoza, Spain

\section{Corresponding author:}

Loriano Galeotti, PhD 


\section{$1 \quad$ Keywords}

2 ECG, simulation, T-wave notch, hERG block, action potential, M-cells

\section{Highlights}

4

\section{$8 \quad$ Structured abstract}

9 Drugs that cause strong hERG potassium channel block (e.g., dofetilide, quinidine) cause T-wave notching. It has been suggested that this is due to prolongation of mid-myocardial (M) cells' action

11 potential duration relative to endocardial and epicardial cells. However, the role of $\mathrm{M}$ cells in intact

12 human hearts is debated.

13 We simulated 2025 electrocardiograms representing changes in ventricular action potentials using the "equivalent double layer model" that does not include M-cells. Action potential changes included "prolongation", "triangularization", "squaring", and "bumps" in late repolarization, which have been observed experimentally and in single cell models with block of the hERG potassium channel. Changes were applied globally and spatially dispersed.

Action potential bumps (slowing in late repolarization) produced T-wave notching similar to that observed clinically in healthy subjects receiving dofetilide or quinidine. Conversely, all other action potential changes (i.e., prolongation, triangularization, squaring), either global or spatially dispersed, 
1 resulted in T-wave changes, but did not cause T-wave notching. This study demonstrates that M-cells 2 are not required to simulate T-wave notching. 


\section{Introduction}

2 Drugs that cause strong block of the human ether-a-go-go related ( $h E R G$ ) potassium ion channel in

3 cardiomyocytes (e.g. dofetilide and quinidine) can cause notched T-waves on the electrocardiogram

4 (ECG). A recent clinical trial studying the effects of dofetilide, quinidine, ranolazine and verapamil in 22

5 healthy volunteers [1] showed the presence of T-wave notches in $64 \%$ of patients receiving dofetilide, a

6 strong hERG potassium channel blocker [2]. Prior preclinical [3] and in silico [4] studies have suggested

7 that extreme prolongation of mid-myocardial (M) cells relative to endocardial and epicardial cells may

8 cause T-wave notching. However, the presence, prevalence and relevance of M-cells in intact human

9 hearts is debated [5]. Thus, it is not clear if T-wave notching can be caused by action potential waveform 10 changes in the absence of M-cells.

11 In this study, we use ECG simulations test the hypothesis that the presence of M-cells is not necessary to

12 cause T-wave notching.

\section{Material and Methods}

14 We simulated 2025 cases of local and global changes of the action potential: triangularization, squaring,

15 prolongation, and repolarization bumps (Figure 1). We applied changes compatible with therapeutic

16 drug concentrations known to cause T-wave notching.

\section{Identification of meaningful action potential changes}

18 We conducted a literature search of published experimental data and simulations of cardiomyocytes to

19 identify meaningful changes in the action potential waveforms. Published experimental data [6]

20 suggested that the action potential changes can be described as a combination of prolongation,

21 triangularization / squaring (change of slope in phase 2 and 3 of the action potential), and terminal

22 repolarization bumps. Using the O'Hara-Rudy model [7], we simulated the effects of a pure-hERG 
1 potassium channel blocking drug (dofetilide) at clinically relevant concentrations (Figure 2). Ion-channel

2 blocking effect was simulated at steady state using the conductance-block model (i.e. by reducing

3 maximal ionic conductance).

4 We simulated dofetilide concentration as $1 \mathrm{nM}, 2 \mathrm{nM}$ (corresponding to the estimated unbound plasma

5 levels observed with a single oral dose of $500 \mu \mathrm{g}$ [2]), $3 \mathrm{nM}$, and $6 \mathrm{nM}$. The corresponding hERG

6 potassium channel block for dofetilide was $8 \%, 30 \%, 36 \%$, and $56 \%$ [2]. In addition, we simulated three

7 multi-channel blocking drugs (quinidine, ranolazine, verapamil) to qualitatively verify what action

8 potential morphological changes are visible when other ion currents (calcium, late-sodium) are blocked

9 in addition to hERG potassium channel. Action potential simulations were visually evaluated to broadly

10 determine a range of action potential waveform modifications to be used in subsequent ECG simulations

11 as described below.

\section{ECG simulation}

13 To simulate ECGs, we used the "equivalent double layer" (EDL) model [8]. This model has been used in

14 multiple peer-reviewed scientific papers [9 - 11] and is implemented in the interactive simulator ECGSIM

15 [12-13]. The EDL model states that the electric potentials at the body surface generated by the heart

16 (e.g., the ECG) are determined completely by the strength of the EDL following the trans-membrane

17 potentials waveform at the surface bounding the active region (the myocardium); thus, this model does

18 not include mid-myocardial M-cells.

19 In this study we used the "normal_young_male" model available with ECGSIM version 3.0.0 [14]. This

20 model is based on ECG and magnetic resonance images of a healthy volunteer (20 years old, $80 \mathrm{~kg}, 188$

$21 \mathrm{~cm})$ and is characterized by 1500 ventricular "nodes" with each one representing a small ( $\approx 0.067 \%)$

22 portion of the ventricular surface. The model includes a transfer function to compute the ECG on the

23 body surface from the node's transmembrane potential or action potential. For each node an action 
1 potential is defined that represent an average cardiomyocytes' action potential for the node area.

2 Multiple parameters control the shape of the action potential waveforms that are constructed as

3 combinations of several sigmoid functions [15]. The depolarization time and repolarization time

4 correspond respectively with the steepest up- and down-slope of the action potential. The difference

5 between depolarization and repolarization times approximately coincides with action potential duration

6 measured at 70\% repolarization. Other two parameters control the slopes of the plateau phase and

7 repolarization phase. The "normal_young_male" model includes appropriate values (referred as

8 "baseline") of action potential parameters (e.g., depolarization time, repolarization time) to simulate an

9 ECG similar to the real ECG from the same volunteer. Baseline action potential parameters were

10 adjusted through an automatic optimization process in order to obtain an ECG as similar as possible to

11 the one recorded on the volunteer.

12 We used MATLAB version R2014b (The MathWorks, Inc., Massachusetts USA) to access ECGSIM data

13 files and simulate ECGs. In particular, a custom MATLAB library allows loading the model files (geometry,

14 volume conductor transfer matrix to determine potentials on torso surface, baseline action potential

15 characteristic for each cardiac node), applying modifications to the action potential waveform and

16 simulating the ECG.

17 All action potential changes described below are applied relative to the baseline value of each node's

18 action potential.

\section{Ranges of variation of action potential waveforms}

20 This study is intended to provide qualitative information (i.e., what change in the action potential, if any,

21 produce T-wave notches), thus we investigated ranges of action potential changes that include those

22 compatible with drug effects. Should be noted that not all the simulated combinations of changes may

23 be realistic, however we believe that these cases may help identifying meaningful patterns. In the ECG 
1 simulator is not possible, at the moment, to directly connect the ECG simulations to cellular

2 electrophysiology (e.g., by directly using AP waveforms from in vitro recordings or generated by the

3 O'Hara-Rudy cardiomyocyte model), but it is however possible to modify the action potential

4 morphology to mimic cellular electrophysiological changes from ion channel block. We focused on

5 morphological changes that have been suggested to be relevant (i.e., bumps, prolongation,

6 triangularization/squaring, spatial dispersion) instead of reproducing exact waveforms. To overcome the

7 uncertainty due the imperfect representation of the action potential changes, we analyzed multiple

8 different levels and combinations of changes, and analyzed the effects that can be observed consistently

9 instead of focusing on simulating only a few specific cases. To highlight the qualitative nature of the

10 study, we labeled the increasing level of action potential changes with a scale from "small", "modest",

11 "moderate" to "large".

\section{Action potential repolarization bumps}

13 We did not find a generally accepted definition of repolarization bumps; however we tried to reproduce

14 the phenomena observable in O'Hara-Rudy simulations (Figure 2) and experimental data [6]. The default

15 action potential waveform model does not include a model of repolarization bumps; however we

16 produced these cases by adding a prolonged version of the action potential. Briefly, repolarization

17 bumps (due to the slowing of the terminal part of repolarization phase) were obtained by combining the

18 original the action potential waveform with a prolonged version of the action potential waveform (i.e.,

19 repolarization time delayed to prolong the action potential duration proportionally to the original

20 value). As an example, to produce a "bump level" of $10 \%$, the original action potential waveform would

21 be scaled by 0.9 , then summed to the action potential prolonged by $10 \%$ and scaled by 0.1 . We

22 simulated four levels of bumps, "small", "modest", "moderate" and "large", corresponding respectively

23 to "bump level" of $10 \%, 20 \%, 30 \%$, and $40 \%$ (Figure $1 \mathrm{~A}$ ). The result is a bump in the terminal part of 
1 repolarization, together with a small prolongation of action potential duration and a slight

2 triangularization.

\section{Action potential prolongation}

4 To simulate action potential prolongation, we did not change depolarization time and modified the

5 repolarization time to prolong the action potential duration proportional to the baseline value. We

6 simulated "small", "modest", "moderate" and "large" prolongation, corresponding to 10\%, 20\%, 30\%

7 and $40 \%$ prolongation of action potential duration (Figure $1 \mathrm{C}$ ). From the plot, it can be noted how large

8 prolongations resulted in slight reductions of the slope in the plateau phase, that correspond to modest

9 squaring of the action potential waveforms.

Action potential triangularization and squaring

11 The ECG simulator has the option to define two slopes for the repolarization phase, one called "plateau

12 slope" to indicate phase 2 of the action potential, and one called "repolarization slope" to indicate

13 phase 3 . We changed these slopes simultaneously to simulate triangularization and squaring. To

14 simulate triangularization, we decreased plateau slope and repolarization slope coefficients relative to

15 the baseline value, while for squaring the slope coefficients were increased. We simulated "small",

16 "modest", "moderate" and "large" triangularization and squaring, corresponding to 10\%, 20\%, 30\% and

$1740 \%$ increase or decrease in the action potential slope coefficients (Figure $1 \mathrm{E}$ and G). We note that

18 traditionally the triangularization of the action potential is assessed by a rough estimate of the slope of

19 phase 3 (e.g., by measuring the time difference between crossing $30 \%$ and $90 \%$ of repolarization [6]).

20 Our simulation of triangularization and squaring also affects the plateau slope (phase 2), as suggested by

21 simulations (Figure 2) and experimental data [6]. 
2 To simulate spatial dispersion of the action potential changes, we defined certain areas where we

3 applied action potential changes while the remaining part of the ventricles was left unchanged. Our

4 simulations included epicardial-only, endocardial-only, and both cardiac surfaces (epicardial +

5 endocardial). A transition area (approximately $2 \mathrm{~cm}$ wide) on the epicardial and endocardial surfaces

6 was maintained between the application areas and the unchanged areas to avoid sharp changes in

7 action potential characteristics. All the changes to the action potential characteristics were applied in a

8 sigmoid-shaped fashion within the transition area. Prolongation and triangularization/squaring were

9 applied with respect to the baseline values, then bumps were applied. We defined nine dispersion

10 patterns based on the description of Opthof et al. [5], characterized by the following application areas in

11 the ventricles: global (all epicardium + all endocardium), left epicardium, right epicardium, apex

12 epicardium, left ventricle endocardium, right ventricle endocardium, anterior epicardium, posterior

13 epicardium, and global epicardium. Each combination of action potential changes was applied to each

14 dispersion pattern.

\section{T-wave notch detection and simulated ECG analysis}

We simulated ECGs corresponding to all the combinations of action potential prolongation of repolarization (no prolongation and 4 levels of prolongation, for a total of 5 cases), repolarization bumps (no bumps and 4 levels of bumps, for a total of 5 cases), triangularization, and squaring (triangularization and squaring are mutually exclusive, thus we simulated 4 levels of triangularization, 4 levels of squaring, and no slope changes, for a total of 9 cases), as well as spatial dispersion patterns (no dispersion and 8 spatial dispersion patterns, for a total of 9). All the possible 2025 combinations of changes were simulated, which can be represented in a 4-dimensional independent variables space. A fifth dimension can represent the dependent variable that quantifies the amount of T-wave notches. T-wave notches usually indicate deviations from the normal smooth progress of a T-wave [16]. The presence of T-wave 
1 notches was determined for each simulated ECG using the "notch score" output of QTGuard+/12SL

2 library (GE Healthcare, United Kingdom). This score is intended to identify observable notches in the

3 ECG, in particular it is 0 when no observable notches are present, and increases up to 1 when notches

4 become observable [16]. R version 3.1.2 (R Foundation for Statistical Computing, Vienna, Austria) was

5 used for exploratory data analysis.

\section{$6 \quad$ Results}

7 We simulated 2025 ECGs representing different changes in the action potential waveforms, and

8 evaluated the notch score of each one. These data can be represented in a five-dimensional space

9 (notch score versus prolongation, triangularization/squaring, bumps, and spatial dispersion). For

10 simplicity we show the effects of globally applied individual action potential changes in Figure 1.

11 Visual analysis of multi-channel blocking drugs' (Quinidine, Ranolazine, Verapamil) O'Hara-Rudy action

12 potential simulations showed that blocking of other ion channel currents tend to mitigate the effects of

13 hERG potassium channel block. However the effect of hERG potassium channel block is not completely

14 canceled by blocking other channels, thus action potential changes such as bumps, prolongation, and

15 triangularization occur for both pure hERG potassium channel block(dofetilide) and drugs with

16 additional inward current block.

17 Simulation of action potential repolarization bumps ("large" and "moderate", Figure $1 \mathrm{~A}$ ) alone was

18 sufficient to cause notches in the T-wave. This was determined by notch score larger than zero and can

19 be visually confirmed by reviewing the simulated ECGs (Figure $1 \mathrm{~B}$, dotted black and solid black lines).

20 Action potential prolongation alone (Figure $1 \mathrm{C}$ ) did not result in T-wave notches (Figure $1 \mathrm{D}$ ). Increasing

21 action potential prolongation resulted in T-wave prolongation and increased amplitude. This amplitude

22 increase is likely due to the associated action potential squaring as described below (Figure $1 \mathrm{G}$ ). 
1 Action potential triangularization alone (Figure $1 \mathrm{E}$ ) did not result in T-wave notches (Figure $1 \mathrm{~F}$ ).

2 Increasing action potential triangularization resulted in T-wave flattening. Action potential squaring

3 alone (Figure $1 \mathrm{G}$ ) did not result in T-wave notches (Figure $1 \mathrm{H}$ ). Increasing action potential squaring

4 resulted in increased T-wave amplitude.

5 Spatial dispersion of action potential prolongation and/or triangularization/squaring did not cause T-

6 wave notches in absence of repolarization bumps. When repolarization bumps were simulated we

7 obtained notches more frequently when applying action potential changes globally ( $44.5 \%$ of cases)

8 compared to all-epicardial (21.6\%), left-ventricle endocardial (3.4\%), right epicardial (2.8\%) left

9 epicardial (2.3\%), apex epicardial (1.1\%) and right-ventricle endocardial (1.1\%). Action potential changes

10 applied only to the anterior or posterior epicardial surfaces never resulted in T-wave notches.

11 Of the 2025 simulated ECGs, we only observed T-wave notches in the presence of repolarization bumps.

12 In some cases, when large repolarization bumps were combined with large prolongation and/or

13 triangularization, the notch score became 0 probably because the T-wave notch detection algorithm

14 identified a secondary T-wave peak as a U wave. A visual comparison between a simulated ECG

15 (baseline and globally applied large bump) and a clinical ECG (baseline and in presence of dofetilide) [2]

16 is shown in Figure 3.

\section{Discussion}

19 This study demonstrates that mid-myocardial cells are not required to simulate T-wave notching. T-wave

20 notching may be caused by morphology changes (bumps) in the late repolarization phase of the cardiac

21 action potential due to hERG potassium channel block. In this study we simulated 2025 ECGs in the

22 absence of mid-myocardial cells. Global "moderate" and "large" action potential repolarization bumps 
1 (slowing in late repolarization) consistently produced T-wave notching (Figure 1) similar to that observed

2 clinically in patients receiving dofetilide (Figure 3). Conversely, other action potential changes (i.e.,

3 prolongation, triangularization, squaring), either alone, combined, globally applied or spatially

4 distributed, resulted in T-wave changes (e.g., changes in T-wave peak location or amplitude), but did not

5 cause T-wave notching.

\section{$6 \quad$ Limitations}

7 A current limitation of the software used to optimize the baseline action potential values requires the

8 use of waveforms obtained as a combination of sigmoids, thus preventing the use of action potential

9 waveforms derived from single-cell cardiomyocyte simulators or in vitro experiments. In this work we

10 attempted to overcome this limitation by determining a range of reasonable changes of the action

11 potential, and simulating ECGs corresponding to various combinations of changes. This did not allow us

12 to directly link a certain simulation to a certain drug effect, however it allowed us to qualitatively study

13 what action potential morphological changes compatible with drug effects result in T-wave notching on

14 the ECG. We believe that a qualitative analysis is appropriate for this study, by pointing out relationships

15 of interests that can help to design future studies that may be able to quantify relationships between

16 cellular-level effects and ECG morphology.

\section{Conclusions}

18 This study suggests that the T-wave notching is a signature of hERG potassium channel block, as it

19 depends on the presence of repolarization bumps. This is consistent with clinical observations in long QT

20 syndrome type 2 patients, who have reduced hERG potassium current and often develop notched T-

21 waves [17], but also with T-wave notches associated with hERG potassium channel blocking drugs such

22 as dofetilide, quinidine and ranolazine [2]. Simulations using the O'Hara-Rudy cellular model indicate

23 that calcium and late sodium current block reduces action potential prolongation generated by hERG 
1 potassium channel block, but does not remove the repolarization bumps. This is congruent with clinical

2 data, showing that T-wave notching is caused both by drugs that only block the hERG potassium channel

3 (e.g., dofetilide) and multi-channel blockers (e.g., quinidine, ranolazine).

4 Traditional measures of action potential triangularization/squaring (usually defined by only two points

5 such as the time between $30 \%$ and $90 \%$ of repolarization) and prolongation alone cannot assess the

6 presence of bumps, thus they may be not adequate to determine the genesis of T-wave notching. This

7 suggests the need to introduce new action potential measurements able to detect and quantify the

8 presence of repolarization bumps, in order to better study in vivo, in vitro and simulated drug effects.

9 This study supports that T-wave notching may be caused by morphological changes in the

10 cardiomyocytes' action potential waveforms, and not by spatial inhomogeneity (dispersion) of action

11 potential prolongation. This is congruent with experiments that showed that ranolazine causes T-wave

12 notching [2] although having small spatial dispersion [18].

\section{Acknowledgements and Disclaimer}

14 This project was supported in part by US Food and Drug Administration (FDA)'s Critical Path Initiative,

15 FDA's Office of Women's Health, and appointments to the Research Participation Program at the Center

16 for Devices and Radiological Health administered by the Oak Ridge Institute for Science and Education

17 through an interagency agreement between the US Department of Energy and FDA. QTGuard+/12SL

18 library was used under a Material Transfer Agreement between GE Healthcare and FDA. No official

19 support or endorsement by FDA is intended nor should be inferred. The mention of commercial

20 products, their sources, or their use in connection with material reported herein is not to be construed

21 as either an actual or implied endorsement of such products by FDA. 


\section{References}

2 [1] Johannesen L, Vicente J, Mason JW, Sanabria C, Waite-Labott K, Hong M, Guo P, Lin J, Sørensen JS,

3

4

5

6

7

Galeotti L, Florian J, Ugander M, Stockbridge N, Strauss DG. Differentiating drug-induced multichannel block on the electrocardiogram: randomized study of dofetilide, quinidine, ranolazine, and verapamil. Clin Pharmacol Ther. 2014 Nov;96(5):549-58. doi: 10.1038/clpt.2014.155. Epub 2014 Jul 23. PubMed PMID: 25054430.

[2] Vicente J, Johannesen L, Mason JW, Crumb WJ, Pueyo E, Stockbridge N, Strauss DG. Comprehensive T wave Morphology Assessment in a Randomized Clinical Study of Dofetilide, Quinidine, Ranolazine, and Verapamil. J Am Heart Assoc. 2015 Apr 13;4(4). pii: e001615. doi: 10.1161/JAHA.114.001615. PubMed PMID: 25870186.

[3] Antzelevitch C. Transmural dispersion of repolarization and the T-wave. Cardiovasc Res. 2001 Jun;50(3):426-31

[4] Xue J, Gao W, Chen Y, Han X. Identify drug-induced T wave morphology changes by a cell-toelectrocardiogram model and validation with clinical trial data. J Electrocardiol. 2009 NovDec;42(6):534-42. doi: 10.1016/j.jelectrocard.2009.07.010. Epub 2009 Aug 22. PubMed PMID: 19700171.

[5] Opthof T, Coronel R, Janse MJ. Is there a significant transmural gradient in repolarization time in the intact heart?: Repolarization Gradients in the Intact Heart. Circ Arrhythm Electrophysiol. 2009 Feb;2(1):89-96. doi: 10.1161/CIRCEP.108.825356. PubMed PMID: 19808447.

[6] Shah RR, Hondeghem LM. Refining detection of drug-induced proarrhythmia: QT interval and TRlaD. Heart Rhythm. 2005 Jul;2(7):758-72. Review. PubMed PMID: 15992736. 
[7] O'Hara T, Virág L, Varró A, Rudy Y. Simulation of the undiseased human cardiac ventricular action potential: model formulation and experimental validation. PLoS Comput Biol. 2011 May;7(5):e1002061. doi: 10.1371/journal.pcbi.1002061. Epub 2011 May 26. PubMed PMID: 21637795; PubMed Central PMCID: PMC3102752.

[8] van Oosterom, A. The Equivalent Double Layer; Source Models for Repolarization. In: Macfarlane PW, van Oosterom A, Pahlm O, Kligfield P, Janse MC, Camm J, editors. Basic Electrocardiology. London: Springer; 2011. p. 227-46.

[9] Galeotti L, Strauss DG, Ubachs JF, Pahlm O, Heiberg E. Development of an automated method for display of ischemic myocardium from simulated electrocardiograms. J Electrocardiol. 2009 MarApr;42(2):204-12. doi: 10.1016/j.jelectrocard.2008.12.007. Epub 2009 Jan 20. PubMed PMID: 19159902.

[10] Galeotti L, van Dam PM, Loring Z, Chan D, Strauss DG. Evaluating strict and conventional left bundle branch block criteria using electrocardiographic simulations. Europace. 2013 Dec;15(12):181621. doi: 10.1093/europace/eut132. Epub 2013 May 23. PubMed PMID: 23703366.

[11] Johannesen L, Vicente J, Gray RA, Galeotti L, Loring Z, Garnett CE, Florian J, Ugander M, Stockbridge $\mathrm{N}$, Strauss DG. Improving the assessment of heart toxicity for all new drugs through translational regulatory science. Clin Pharmacol Ther. 2014 May;95(5):501-8. doi: 10.1038/clpt.2013.238. Epub 2013 Dec 12. PubMed PMID: 24336137.

[12] van Oosterom A, Oostendorp TF. ECGSIM: an interactive tool for studying the genesis of QRST waveforms. Heart 2004;90:165-8. doi:10.1136/hrt.2003.014662

[13] van Dam P, van Oostendorp T. Interactive Simulation of the Activation Sequence: replacing effect by cause. Comput Cardiol 2011; $38: 657-60$. 
[14] www.ecgsim.com - Last accessed May 7, 2015

2 [15] van Dam PM, Oostendorp TF, Linnenbank AC, van Oosterom A. Non-invasive imaging of cardiac activation and recovery. Annals Biomedical Engineering. 2009;37(9):1739-56.

4 [16] Andersen MP, Xue JQ, Graff C, Kanters JK, Toft E, Struijk JJ. New descriptors of T-wave morphology

5 are independent of heart rate. J Electrocardiol. 2008 Nov-Dec;41(6):557-61. doi: 10.1016/j.jelectrocard.2008.07.021. Epub 2008 Sep 19. PubMed PMID: 18804787.

[17] Moss AJ, Zareba W, Benhorin J, Locati EH, Hall WJ, Robinson JL, Schwartz PJ, Towbin JA, Vincent GM, Lehmann MH. ECG T-wave patterns in genetically distinct forms of the hereditary long QT syndrome. Circulation. 1995 Nov 15;92(10):2929-34. PubMed PMID: 7586261.

11 L, Milberg P. New insights into the beneficial electrophysiologic profile of ranolazine in heart failure:

12 prevention of ventricular fibrillation with increased postrepolarization refractoriness and without drug-

13 induced proarrhythmia. J Card Fail. 2012 Dec;18(12):939-49. doi: 10.1016/j.cardfail.2012.10.017.

14 PubMed PMID: 23207083. 

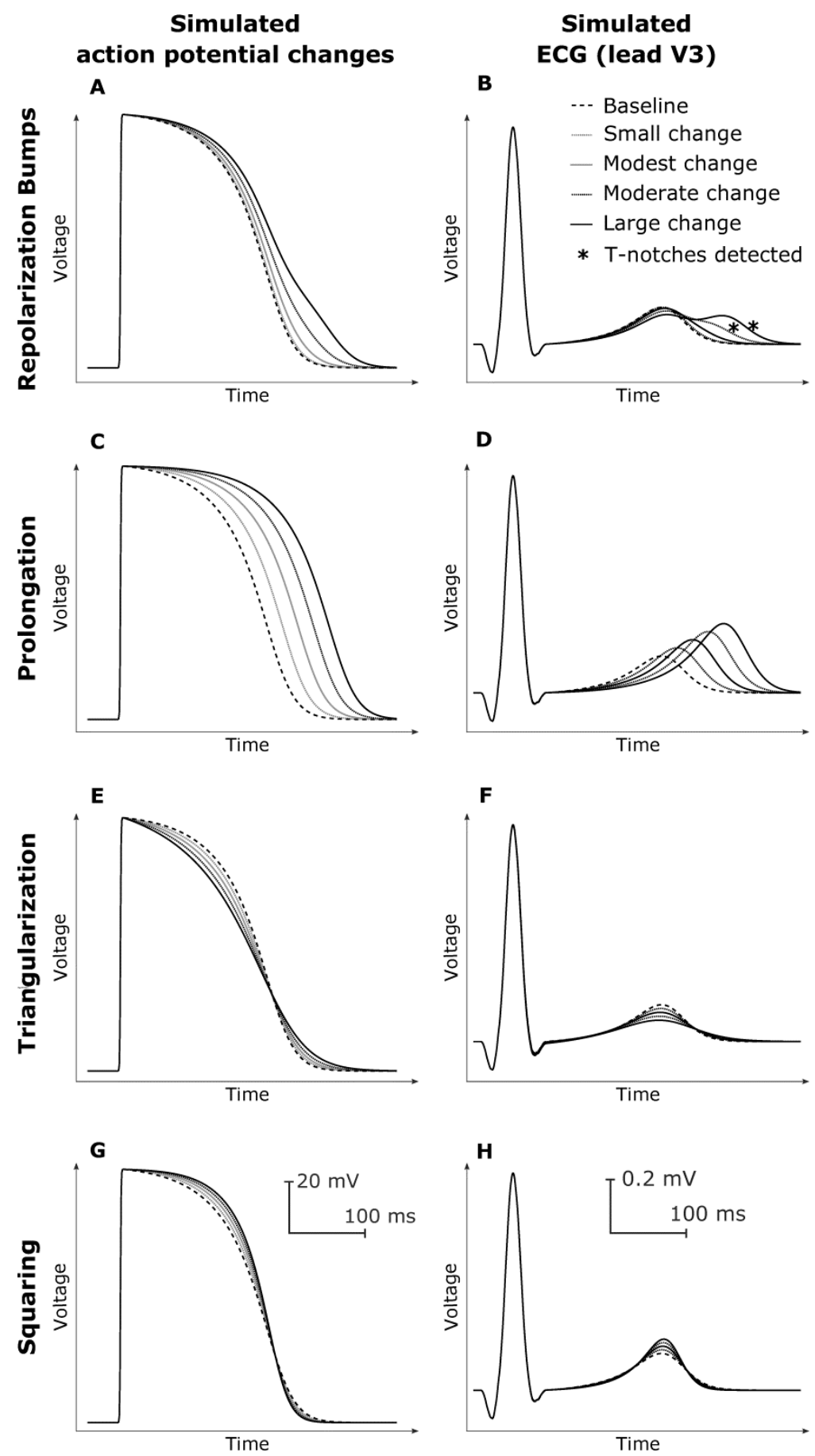

2 Figure 1: Effect of changes in action potential on the ECG (lead V3). Each horizontal pair of plots (A-B, C$D, E-F, G-H)$ corresponds to a simulated action potential change (repolarization bumps, prolongation,

4 triangularization, squaring) and corresponding ECG. In each plot the black-dotted line corresponds to the 5 baseline value, while increased darkness (from gray to solid black) corresponds to small, modest, 
1 moderate and large action potential changes. Asterisk (*) denotes ECGs where T-wave notches were

2 detected (moderate and large repolarization bumps).

3

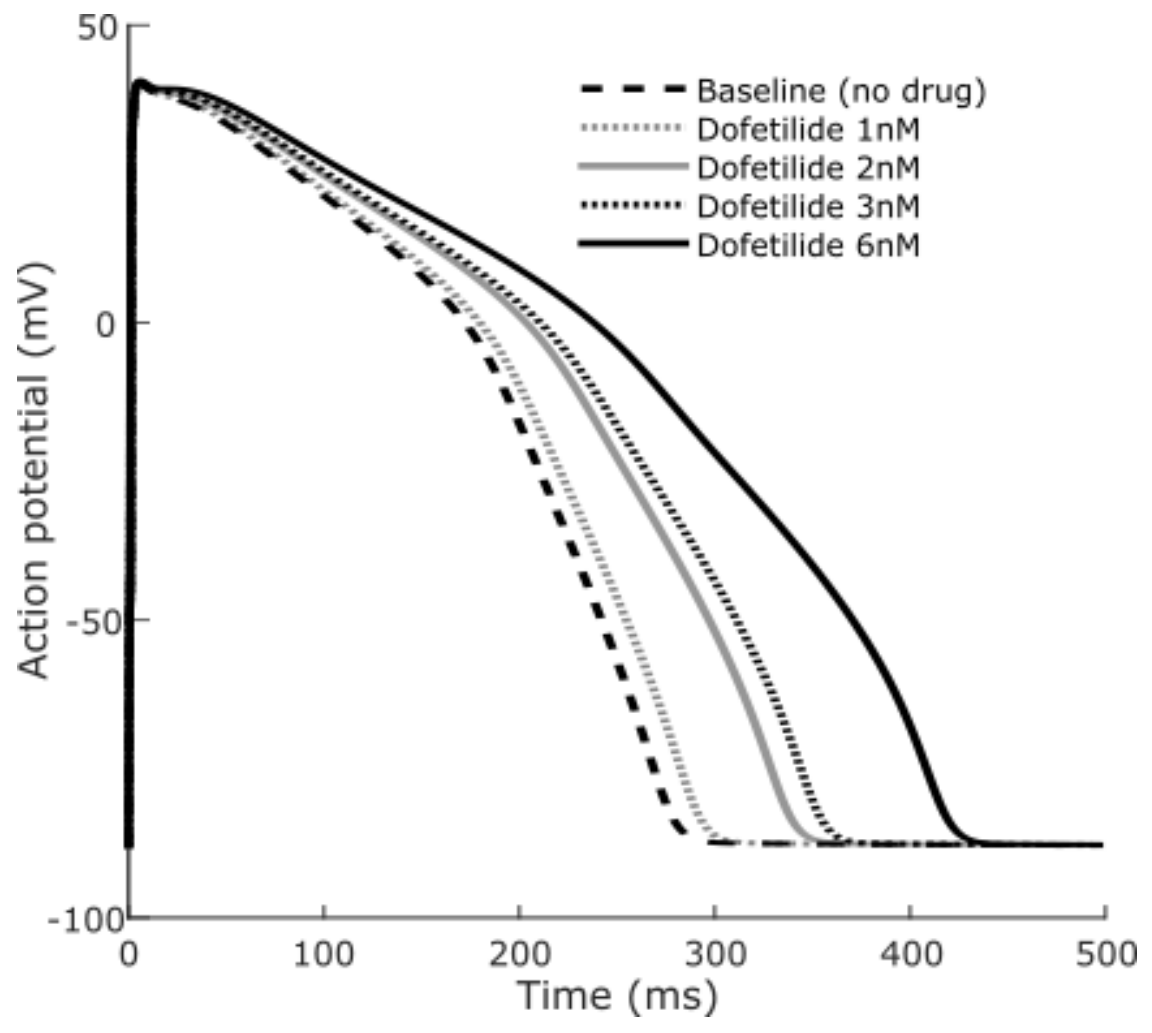

$5 \quad$ Figure 2: Simulations of the effect of various concentrations of dofetilide on the action potential waveform of an endocardial cardiomyocyte, obtained using the O'Hara-Rudy model. 2nM concentration

7 (solid gray line) corresponds to a clinically relevant concentration. Note the presence of bumps (changes

8 in slope) in the terminal part of repolarization evident at $2 \mathrm{nM}$ and higher drug concentration. 

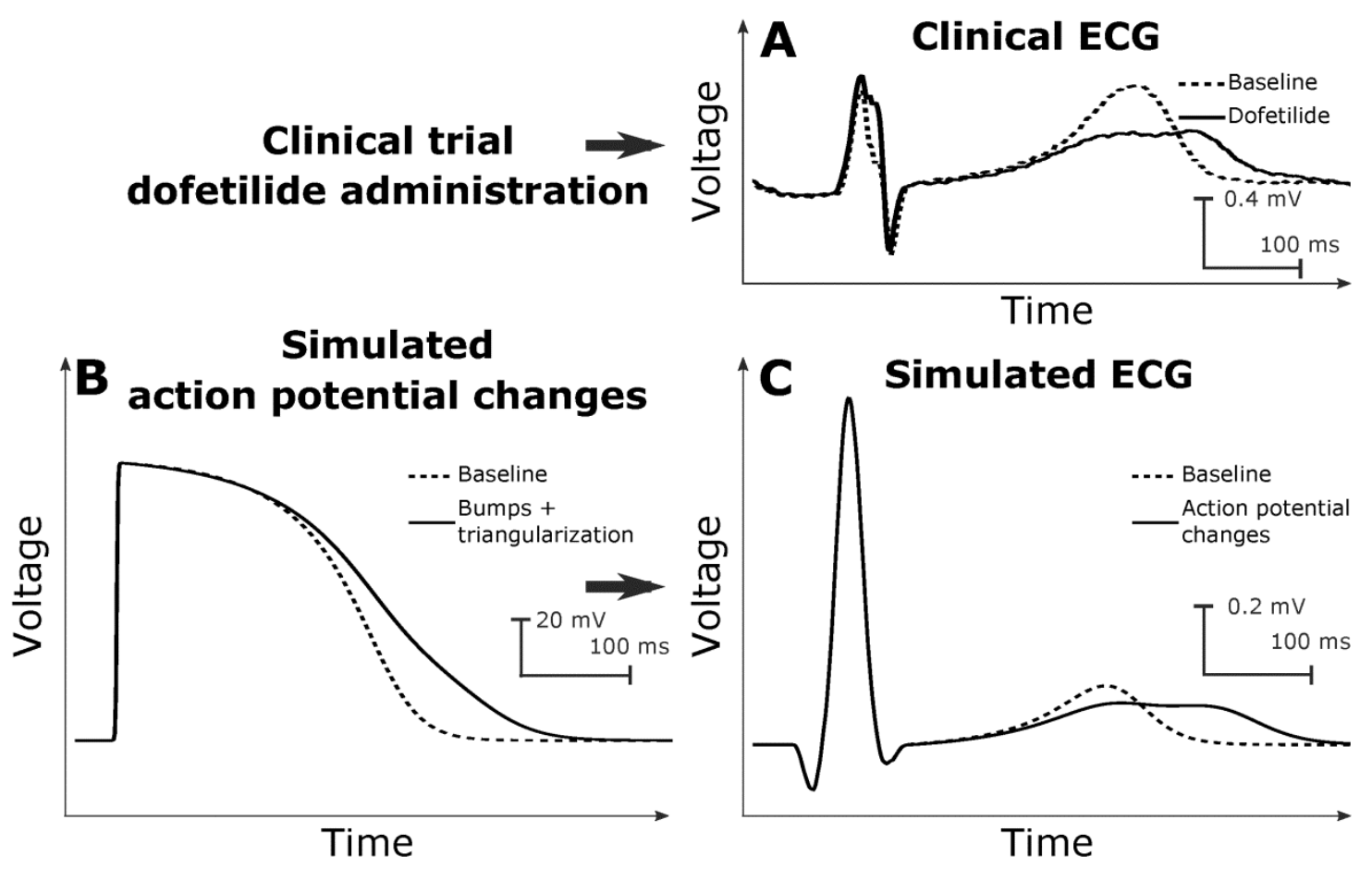

2 Figure 3: Qualitative comparison of the effects on the ECG (lead V3) of hERG potassium blocking drug

3 dofetilide (panel A, from Vicente et al. [2]) and simulated action potential changes (large repolarization

4 bumps + modest triangularization). Panel B shows action potential waveform; panel C shows the

5 corresponding simulated ECG. Both clinical and simulated ECGs show T-wave notches and flattening. 


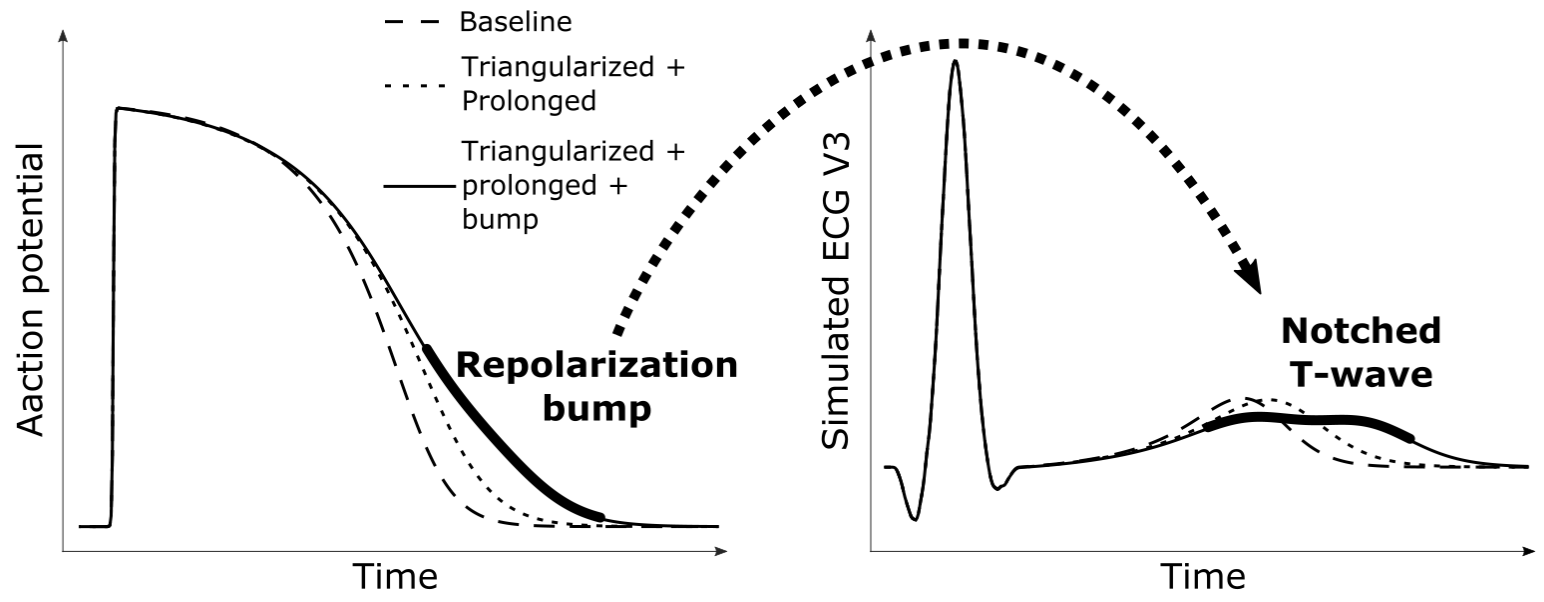

2 Graphical abstract 


\section{Figưfe 2}

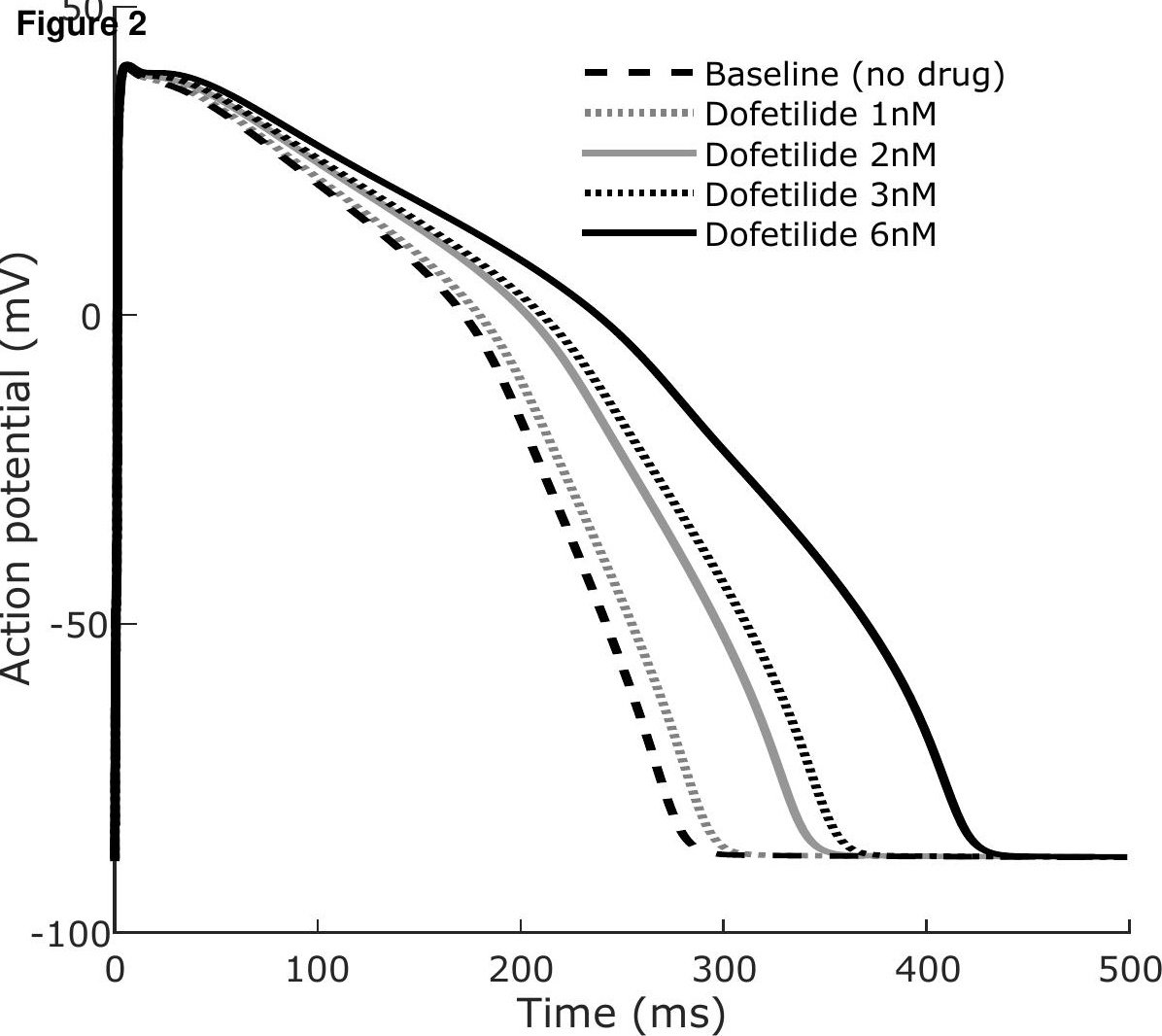


